\title{
Upset Recovery Control for Quadrotors Subjected to a Complete Rotor Failure from Large Initial Disturbances
}

\author{
Sihao Sun*, Matthias Baert*, Bram Strack van Schijndel and Coen de Visser
}

\begin{abstract}
This study has developed a fault-tolerant controller that is able to recover a quadrotor from arbitrary initial orientations and angular velocities, despite the complete failure of a rotor. This cascaded control method includes a position/altitude controller, an almost-global convergence attitude controller, and a control allocation method based on quadratic programming. As a major novelty, a constraint of undesirable angular velocity is derived and fused into the control allocator, which significantly improves the recovery performance. For validation, we have conducted a set of Monte-Carlo simulation to test the reliability of the proposed method of recovering the quadrotor from arbitrary initial attitude/rate conditions. In addition, real-life flight tests have been performed. The results demonstrate that the post-failure quadrotor can recover after being casually tossed into the air.
\end{abstract}

\section{INTRODUCTION}

In recent years, multi-rotor aerial vehicles have received a lot of attention. These aerial vehicles are usually unmanned robots that can perform various tasks, in some cases without human intervention. Multi-rotors are mainly used outdoors for agricultural purposes, architecture and construction, delivery, emergency services, media purposes or to monitor and conserve the environment. As these vehicles will become more involved in daily life, safety can not be overlooked.

One of the most common multi-rotors is the quadrotor due to its simplicity and energy efficiency [1]. As the name implies, a quadrotor has four rotors positioned in a rectangular profile on the vehicle. However, because this vehicle is not over-actuated, this type of multi-rotor suffers most from an actuator failure and might not be able to continue its mission or worse, might not be able to land safely.

\section{A. Fault-Tolerant Control}

Fault-tolerant control (FTC) for quadrotors has been the subject of various literature sources. Some research is focused on the partial damage of a rotor [2], [3], while other research considers the complete loss of one or multiple rotors. A solution to the case of a complete loss of a rotor is presented in [4] where the author proposes to give up on yaw control to maintain control over the other states. An analytical solution under the complete loss of one, two or three propellers are given in [5], [6]. A PID and a backstepping approach focusing on an emergency landing in case of failure is presented in [7], [8] respectively. A

*These two authors contributed equally

The authors are with Faculty of Aerospace Engineering, Delft University of Technology, 2629 HS Delft, The Netherlands. email: s.sun-4@ tudelft.nl; c.c.devisser@tudelft.nl

The video is available at: https://youtu.be/PJ5U3ZAm8NM

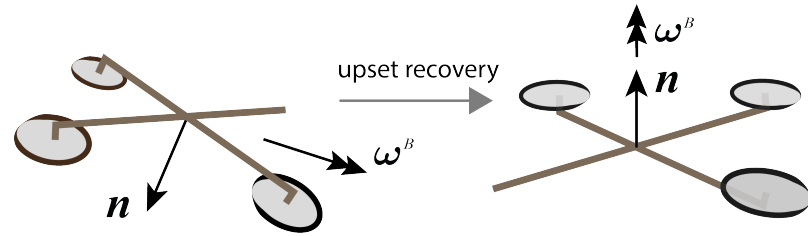

Fig. 1: Illustration of the upset recovery problem where $\boldsymbol{n}$ indicates the total thrust direction and $\omega^{B}$ indicates the vehicle angular velocity.

fault-tolerant controller using incremental nonlinear dynamic inversion (INDI) is given in [9] where fault detection is also implemented. To improve the robustness of the controller, [10] employs a nonsingular terminal sliding mode control (NTSMC) to this fault-tolerant control problem.

The validations in practice are carried out by [5] using the linear quadratic regulator (LQR) around the proposed analytical equilibrium. To improve the stability under various yaw rates, the study in [11] employs a linear parameter varying (LPV) controller. In [12], a quadrotor with loss of single rotor controlled by INDI is shown able to fly in high-speed conditions despite significant aerodynamic disturbances.

\section{B. Upset Recovery}

Upset recovery is a technique extensively studied for improving aviation safety [13], [14]. The upset condition is defined as "any uncommanded or inadvertent event with an abnormal aircraft attitude, angular rate, acceleration, airspeed, or flight trajectory [15]", such as aircraft stall that directly leads to loss-of-control [16]. In comparison, upset of a multi-rotor drone is rarely heard by virtue of its relatively high control effectiveness in full flight envelope. For example, a quadrotor can easily perform aerobatic maneuvers [17].

However, due to the significant maneuverability reduction, a quadrotor with single-rotor-failure can easily enter an upset condition. For instance, as Fig. 1] shows, a post-failure quadrotor may be upside down and fast rotating before the FTC is triggered, because of strong wind disturbances and delay of the fault detection module. At this moment, existing FTC methods could fail owing to multiple reasons, such as violation of linearization assumptions, actuator saturations, etc. Therefore, an improved FTC method is required to address the upset recovery problem.

\section{Contributions}

As the main contribution, this research proposes a controller which has the ability to recover a quadrotor with 


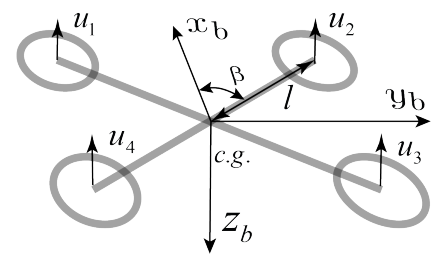

Fig. 2: Definition of the body frame, the geometric parameters $\beta$ and $l$, the index of control inputs.

complete loss of a rotor from an arbitrary attitude and a wide range of initial angular velocities. Then the method can subsequently steer the damaged drone to a designated position and altitude. This cascaded control method is composed of three parts: a control allocator that tracks the angular acceleration command while suppressing the undesirable angular rate, an attitude controller with an almost-global attraction region, and a position controller subordinate to the former two parts.

The control method has been validated in a real-life environment where the quadrotor was randomly tossed into the air and recovers thereafter. A set of Monte-Carlo simulations have been also performed to test the performance of the controller from a wide range of initial conditions. It is shown that the proposed method can significantly improve the quadrotor safety after rotor failures despite large initial disturbances.

\section{PROBLEM Formulation}

\section{A. Notation}

The inertial frame is represented by the north-east-down coordinate system. The body frame is originated at the c.g. of the vehicle with the forward-right-down convention, as is shown in Fig. 2. Throughout the paper, we use lowercase boldface symbols to denote vectors, upper-case boldface symbols for matrices and non-boldface symbols for scalars. A 3-D vector with superscript $[\cdot]^{B}$ indicates that the vector is expressed in the body frame, otherwise in the inertial frame. Operator $\operatorname{diag}(\cdot)$ indicates a diagonal matrix with element $(\cdot)$ as diagonal entries.

\section{B. 6-DoF Model of a Quadrotor}

The quadrotor is powered by four independently controlled rotors to produce necessary lift and control moments. Fig. 2 shows the definition of the body frame, and the rotor index of a quadrotor. The state equations of a quadrotors can be composed of the following 6-DoF rigid body kinematics and dynamic equations [18]:

$$
\begin{gathered}
\dot{\boldsymbol{\xi}}=\boldsymbol{v} \\
\dot{\boldsymbol{R}}=\boldsymbol{R} \omega_{\times}^{B} \\
m \dot{\boldsymbol{v}}=m \boldsymbol{g}+\boldsymbol{R}\left(\boldsymbol{f}_{c}^{B}+\boldsymbol{f}_{a}^{B}\right) \\
\boldsymbol{I}_{v}^{B} \dot{\boldsymbol{\omega}}^{B}+\omega^{B} \times \boldsymbol{I}_{v}^{B} \omega^{B}=\boldsymbol{m}_{c}^{B}+\boldsymbol{m}_{a}^{B}+\boldsymbol{m}_{g}^{B}
\end{gathered}
$$

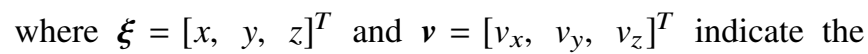
position and velocity respectively. $\boldsymbol{R} \in \mathrm{SO}(3)$ is the rotational matrix of the quadrotor from the body frame to the inertial frame. Therefore, for any vector $\boldsymbol{e} \in \mathbb{R}^{3}$, we have $\boldsymbol{e}=\boldsymbol{R} \boldsymbol{e}^{B}$. The angular velocity of the body frame w.r.t the inertial frame is expressed as $\omega^{B}=\left[\omega_{x}, \omega_{y}, \omega_{z}\right]^{T}$, where $\omega_{\times}^{B}$ is the skew symetric matrix such that $\omega_{\times} \boldsymbol{a}=\boldsymbol{\omega} \times \boldsymbol{a}$ for any $\boldsymbol{a} \in \mathbb{R}^{3}$. The vehicle mass and inertia are denoted by $m$ and $\boldsymbol{I}_{\boldsymbol{v}}{ }^{B}$ respectively and $\boldsymbol{g}$ denotes the gravity vector. The control forces $\boldsymbol{f}_{c}^{B}$ and moments $\boldsymbol{m}_{c}^{B}$ are produced by rotors. A simplified model of forces and moments generated by rotors are expressed as

$$
\boldsymbol{f}_{c}^{B}=\left[0,0, \boldsymbol{G}_{t} \boldsymbol{u}\right]^{T}, \quad \boldsymbol{m}_{c}^{B}=\boldsymbol{G}_{m} \boldsymbol{u}
$$

where $\boldsymbol{u}=\left[u_{1}, u_{2}, u_{3}, u_{4}\right]^{T}$ and $u_{i}$ is the force produced by rotor $i$ (see Fig. 22). Note that $0 \leq \boldsymbol{u}_{\min } \leq \boldsymbol{u} \leq \boldsymbol{u}_{\max }$. When complete failure of rotor $i$ occurs, we have $u_{\min , i}=u_{\mathrm{max}, i}=$ 0. $\boldsymbol{G}_{m}$ is a mapping from rotor generated forces to control moments and $\boldsymbol{G}_{t}$ is the mapping from rotor generated forces to the total thrust. For a quadtrotor that the thrust of each rotor is parallel with the $z_{b}$ axis, we have

$$
\begin{gathered}
\boldsymbol{G}_{t}=[-1,-1,-1,-1] \\
\boldsymbol{G}_{m}=\operatorname{diag}(l \sin \beta, l \cos \beta, s \sigma)\left[\begin{array}{cccc}
1 & -1 & -1 & 1 \\
1 & 1 & -1 & -1 \\
1 & -1 & 1 & -1
\end{array}\right]
\end{gathered}
$$

where $l$ and $\beta$ are geometric parameters as shown in Fig. 2 . $\sigma$ is the torque thrust ratio of the rotor, $s$ is a sign variable determined by the rotating direction of the rotor.

The force model given by (5) neglects the variation of thrust stem from quadrotor translational motions with respect to the airflow. Therefore, an aerodynamic force term $\boldsymbol{f}_{a}^{B}$ is added in (3), so as the term $\boldsymbol{m}_{a}^{B}$ in (4). The gyroscopic moment, denoted by $\boldsymbol{m}_{g}^{B}$, is caused by the rotation of rotors with respect to the body frame. For the current research, we omit $\boldsymbol{f}_{a}^{B}, \boldsymbol{m}_{a}^{B}$ and $\boldsymbol{m}_{g}^{B}$ in the controller design whereas they are included in the simulation presented in Sec IV

\section{Quadrotor Upset Recovery Problem}

A quadrotor has four independently powered rotors, such that the thrust, pitch, roll and yaw channels can be totally decoupled. This characteristic, however, can be different when a single rotor failure occurs. A most commonly used strategy is by giving up the yaw control and keep the rest which is more essential for maintaining the desired position and altitude [4]. This requires the post-failure vehicle to enter a so-called relaxed-hovering condition [6] in which the drone spins about an average thrust direction whilst the position of the spinning center and the altitude maintain constant. By slightly changing the direction and amount of the reference thrust, the average position and altitude of the post-failure quadrotor can be controlled.

Driving a quadrotor with a single rotor failure to the relaxed-hovering condition from arbitrary initial attitude, angular rates, and positions, is defined as the quadrotor upset recovery problem. 


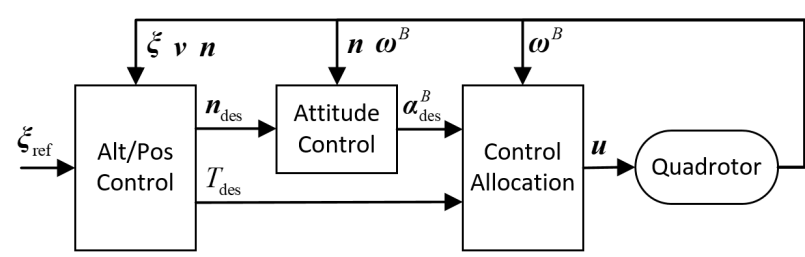

Fig. 3: Diagram of the proposed control method.

\section{Methodology}

The major challenge of the recovery problem is threefold. First of all, we need to design an almost-global (excluding finite singularities) reduced attitude controller to drive the vehicle orientation to the relaxed-hovering condition from large initial attitude deviation. Secondly, with the complete failure of a rotor, the quadrotor system only has three remaining constraint inputs. Hence it requires a novel control allocation approach to address input constraints while preventing the drone from entering upset conditions. Last but not least, a hedging of position/altitude loop need to be designed to coordinate with the aforementioned attitude controller and the control allocation method. A cascaded framework of the proposed controller is given as Fig. 3 shows.

\section{A. Altitude and Position Control}

The position and altitude control, namely the outer-loop control, is designed as a cascaded P+PI controller as follows

$$
\begin{gathered}
\boldsymbol{v}_{\mathrm{des}}=\boldsymbol{K}_{p, \text { pos }}\left(\boldsymbol{\xi}_{\text {ref }}-\boldsymbol{\xi}\right) \\
\boldsymbol{a}_{\mathrm{des}, 0}=\boldsymbol{K}_{p, \text { vel }}\left(\boldsymbol{v}_{\mathrm{des}}-\boldsymbol{v}\right)+\boldsymbol{K}_{i, \mathrm{vel}} \int\left(\boldsymbol{v}_{\mathrm{des}}-\boldsymbol{v}\right) \mathrm{d} t-\boldsymbol{g}
\end{gathered}
$$

where $\boldsymbol{\xi}_{\text {ref }}$ is the reference position; $\boldsymbol{K}_{p, \text { pos }}, \boldsymbol{K}_{p \text {,vel }}$ and $\boldsymbol{K}_{i \text {,vel }}$ are $3 \times 3$ positive diagonal gain matrices. The acceleration reference is then obtained by

$$
\boldsymbol{a}_{\mathrm{des}}=\operatorname{diag}(1 / \epsilon, 1 / \epsilon, 1) \boldsymbol{a}_{\mathrm{des}, 0}
$$

where

$$
\epsilon=\max \left(\sqrt{a_{x, \text { des }, 0}^{2}+a_{y, \text { des }, 0}^{2}} / a_{z, \text { des }, 0} \tan \theta_{1}, 1\right)
$$

Then we can obtain the desired thrust direction

$$
\boldsymbol{n}_{\mathrm{des}}=\boldsymbol{a}_{\mathrm{des}} /\left\|\boldsymbol{a}_{\mathrm{des}}\right\|
$$

Note that the transform (10) guarantees that the angle between $\boldsymbol{n}_{\mathrm{des}}$ and the reverse of gravity $-\boldsymbol{g}$ is confined by angle $\theta_{1}$. Limiting this desired thrust direction can prevent aggressive spatial maneuvers during recovery.

Now we use $\theta$ to denote the angle between current thrust direction $\boldsymbol{n}$ and $-\boldsymbol{g}$

$$
\theta=\arccos (-\boldsymbol{g} \cdot \boldsymbol{n} /\|\boldsymbol{g}\|)
$$

Then the original thrust command can be obtained by

$$
T_{\text {des }, 0}=-m \cdot a_{z, \text { des }} / \cos \theta
$$

However, this method may deteriorate the attitude loop performance. Consider when the drone is upside down where $\theta \geq 90 \mathrm{deg}$, 14 gives a negative thrust command; or when $\theta=90 \mathrm{deg}$, (14) leads to singularity. For this reason, a scaling factor $\beta$ is introduced which is scheduled by the total incline angle $\theta$, yielding

$$
\beta=\frac{\theta_{2}-\min \left(\max \left(\theta, \theta_{1}\right), \theta_{2}\right)}{\theta_{2}-\theta_{1}}
$$

where $\theta_{1}<\theta_{2}$ are predetermined parameters (see Table. II). Finally the total thrust command is obtained by

$$
T_{\text {des }}=-\beta \cdot m \cdot a_{z, \text { des }} / \cos \left(\min \left(\theta, \theta_{1}\right)\right)
$$

\section{B. Attitude Control}

The attitude controller calculates the the angular rate command in order to control the thrust orientation $\boldsymbol{n}$ to $\boldsymbol{n}_{\mathrm{des}}$. Now introduce the total incline angle $\rho$ as the angle from $\boldsymbol{n}_{\text {des }}$ to $\boldsymbol{n}$, where

$$
\rho=\arccos \left(\boldsymbol{n}_{\text {des }} \cdot \boldsymbol{n}\right)
$$

Define the instant rotation vector $\boldsymbol{n}_{c}$ perpendicular to both $\boldsymbol{n}_{\text {des }}$ and $\boldsymbol{n}$, we have

$$
\boldsymbol{n}_{c}=\boldsymbol{n} \times \boldsymbol{n}_{\mathrm{des}} / \sin \rho
$$

The reference angular rate can be consequently obtained

$$
\boldsymbol{\omega}_{\mathrm{des}}^{B}=k_{p, \text { att }} \cdot \rho \boldsymbol{R}^{T} \boldsymbol{n}_{c}
$$

where $k_{p \text {,att }}$ is a positive gain. Then the angular acceleration reference can be obtained by a proportional controller with a feed-forward term

$$
\alpha_{\text {des }}^{B}=K_{p, \text { rate }}\left(\omega_{\text {des }}^{B}-\omega^{B}\right)+\dot{\omega}_{\text {des }}^{B}
$$

Note that (18) becomes singular when $\boldsymbol{n}$ and $\boldsymbol{n}_{\mathrm{des}}$ are collinear. Thus the attitude control presented above could result in the almost-global reduced attitude stabilization [19] with exception of two special points, namely $\rho \in\{0, \pi\}$. In practice, when singularity occurs, we can simply set $\boldsymbol{n}_{c}$ as an arbitrary unit vector perpendicular to $\boldsymbol{n}$.

\section{Control Allocation}

The control allocation step solves the desired thrust of each rotor, namely $\boldsymbol{u}$, using the desired angular acceleration $\alpha_{\text {des }}^{B}$ and the total thrust command $T_{\text {des }}$ as calculated above. Now, we use $\mu_{\text {des }}$ to denote the desired control moments and thrust. By replacing $\dot{\omega}^{B}$ with $\boldsymbol{\alpha}_{\mathrm{des}}^{B}$ in 4 and omitting $\boldsymbol{m}_{g}$ and $\boldsymbol{m}_{a}$, we have

$$
\boldsymbol{\mu}_{\mathrm{des}}=\left[\begin{array}{c}
\boldsymbol{m}_{c, \mathrm{des}}^{B} \\
T_{\mathrm{des}}
\end{array}\right]=\left[\begin{array}{c}
\boldsymbol{I}_{v}^{B} \boldsymbol{\alpha}_{\mathrm{des}}^{B}+\boldsymbol{\omega}^{B} \times \boldsymbol{I}_{v}^{B} \boldsymbol{\omega}^{B} \\
T_{\mathrm{des}}
\end{array}\right]
$$

The thrusts generated by rotors need to cooperatively fulfil the reference represented by $\boldsymbol{\mu}_{\mathrm{des}}$. As the thrust produced by a rotor is limited, we establish a constrained Quadratic Programming (QP) problem to solve $\boldsymbol{u}$ :

$$
\begin{array}{cc}
\text { P1 : } & \min _{\substack{\boldsymbol{u} \\
\text { s.t. }}}\left(\boldsymbol{\mu}_{\mathrm{des}}-\boldsymbol{G} \boldsymbol{u}\right)^{T} \boldsymbol{W}\left(\boldsymbol{\mu}_{\mathrm{des}}-\boldsymbol{G} \boldsymbol{u}\right)+\lambda \boldsymbol{u}^{T} \boldsymbol{u} \\
& \boldsymbol{u}_{\min } \leq \boldsymbol{u} \leq \boldsymbol{u}_{\max }
\end{array}
$$

where $\boldsymbol{G}=\left[\boldsymbol{G}_{m}^{T}, \boldsymbol{G}_{t}^{T}\right]^{T}$ is a combined control effective matrix; $\boldsymbol{W}=\operatorname{diag}\left(W_{x}, W_{y}, W_{z}, W_{t}\right)$ is a user defined weighting matrix, which determines the weight for each control 


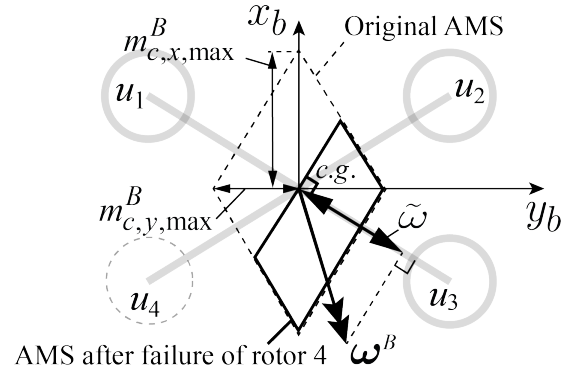

Fig. 4: Projection of the attainable moment set (AMS) on the $x_{b}-y_{b}$ plane before and after the failure of rotor 4 . The projection of current angular velocity $\omega^{B}$ perpendicular to the boundary of AMS is unable to be reduced by the control moment. The magnitude of this component is denoted by $\tilde{\omega}$.

objective; $\lambda>0$ is another weight for minimizing the control effort.

P1 is a typical control allocation method for both aircraft and drones [20], [21]. However, for a quadrotor with single rotor failure, we need to add an additional constraint to P1. We hereby define the Attainable Moment Set (AMS) as a set of moments that can be generated by the existing rotors. As Fig. 4 shows, the area of AMS is reduced after rotor failure occurs. This is due to the fact that the quadrotor with fixed-pitch rotors can not generate negative lift, namely $\boldsymbol{u}_{\text {min }} \geq 0$. In consequence, the angular velocity which cannot be suppressed by the current attainable moment will cause unstoppable rotations. The magnitude of this angular velocity, denoted by $\tilde{\omega}$, must be restrained during upset recovery.

A constraint of $\tilde{\omega}$ after a brief time period $t_{h}$ is then introduced. Since the maneuverability on pitch/roll direction is much higher than yaw direction, we assume that $\omega_{z}$ in the period $t_{h}$ is constant. Recall (4), and approximate $I_{v}^{B}$ by $\operatorname{diag}\left(I_{x}, I_{y}, I_{z}\right)$, we have

$$
\left[\begin{array}{c}
\dot{\omega}_{x} \\
\dot{\omega}_{x}
\end{array}\right]=\left[\begin{array}{cc}
0 & \frac{I_{y}-I_{z}}{I_{x}} \omega_{z} \\
\frac{I_{z}-I_{x}}{I_{y}} \omega_{z} & 0
\end{array}\right]\left[\begin{array}{c}
\omega_{x} \\
\omega_{y}
\end{array}\right]+\hat{\boldsymbol{G}}_{m} \boldsymbol{u}
$$

where

$$
\hat{\boldsymbol{G}}_{m}=\operatorname{diag}\left(I_{x}, I_{y}\right)^{-1}\left[\begin{array}{lll}
1 & 0 & 0 \\
0 & 1 & 0
\end{array}\right] \boldsymbol{G}_{m}
$$

Note that 23 is a linear ODE, thus the time history of $\omega_{x}$ and $\omega_{y}$ can be explicitly solved with given initial conditions and control inputs. Assume the control input $\boldsymbol{u}$ is constant within $t_{h}$, and use the current $\omega_{x}$ and $\omega_{y}$ as initial conditions, then $\tilde{\omega}$ after $t_{h}$ can be expressed as

$$
\tilde{\omega}\left(t_{h}\right)=\boldsymbol{\phi}\left[\begin{array}{c}
\omega_{x}\left(t_{h}\right) \\
\omega_{y}\left(t_{h}\right)
\end{array}\right]=\boldsymbol{\phi} \boldsymbol{\Phi}_{0}\left(t_{h}\right)\left[\begin{array}{c}
\omega_{x} \\
\omega_{y}
\end{array}\right]+\boldsymbol{\phi} \boldsymbol{\Phi}_{1}\left(t_{h}\right) \hat{\boldsymbol{G}}_{m} \boldsymbol{u}
$$

where $\phi \in \mathbb{R}^{1 \times 2}$ is a row vector converting $\omega_{x}$ and $\omega_{y}$ to $\tilde{\omega}$, and we have

$$
\begin{gathered}
\boldsymbol{\Phi}_{0}=\left[\begin{array}{cc}
\cos \left(c t_{h}\right) & -\frac{c}{b} \sin \left(c t_{h}\right) \\
\frac{b}{c} \sin \left(c t_{h}\right) & \cos \left(c t_{h}\right)
\end{array}\right] \\
\boldsymbol{\Phi}_{1}=\left[\begin{array}{cc}
\frac{1}{c} \sin \left(c t_{h}\right) & \frac{1}{b} \cos \left(c t_{h}\right)-\frac{1}{b} \\
\frac{b}{c^{2}}-\frac{b}{c^{2}} \cos \left(c t_{h}\right) & \frac{1}{c} \sin \left(c t_{h}\right)
\end{array}\right]
\end{gathered}
$$

where

$$
b=\frac{I_{z}-I_{x}}{I_{y}} \omega_{z}, \quad c=\sqrt{\frac{\left|\left(I_{z}-I_{x}\right)\left(I_{y}-I_{z}\right)\right|}{I_{x} I_{y}}}\left|\omega_{z}\right|
$$

Note that the detail expression of $\phi$ varies with the quadrotor geometric property and the location of the failure rotor in the body frame.

From 25 , it is clear that $\tilde{\omega}$ is not only affected by the rotor generated moments, but also coupling moment (term $\omega^{B} \times I_{v}^{B} \omega^{B}$ in $(4)$ ) as the function of initial angular velocity. Therefore, reducing $\tilde{\omega}$ is possible by leveraging these coupling moments after complete failure of rotors.

Consequently, the control allocation method constraining $\tilde{\omega}$ can be constructed as

$$
\begin{array}{cc}
\mathrm{P} 2: \min _{\boldsymbol{u}, d} & \left(\boldsymbol{\mu}_{\mathrm{des}}-\boldsymbol{G} \boldsymbol{u}\right)^{T} \boldsymbol{W}\left(\boldsymbol{\mu}_{\mathrm{des}}-\boldsymbol{G} \boldsymbol{u}\right)+\lambda \boldsymbol{u}^{T} \boldsymbol{u}+\gamma d^{2} \\
\text { s.t. } \quad \boldsymbol{\phi} \boldsymbol{\Phi}_{1} \hat{\boldsymbol{G}}_{m} \boldsymbol{u} \leq-\boldsymbol{\phi} \boldsymbol{\Phi}_{0}\left[\omega_{x}, \omega_{y}\right]^{T}+\tilde{\omega}_{\max }+d \\
\quad-d \leq 0 \\
\boldsymbol{u}_{\min } \leq \boldsymbol{u} \leq \boldsymbol{u}_{\max }
\end{array}
$$

where the first constraint stems from (25), which sets limitations to the $\tilde{\omega}$ after $t_{h}$ by $\tilde{\omega}_{\max }$. The slack variable $d$ is added to guarantee the solution of above optimization problem; $\gamma>0$ is a weight added to the slack variable thereof. Note that the recovery performance is affected by three parameters: $t_{h}, \tilde{\omega}_{\max }$ and $\gamma$. In general, the constraint of $\tilde{\omega}$ is more strict with a larger $t_{h}, \gamma$ and a smaller $\tilde{\omega}_{\max } . \mathrm{P} 2$ is a constrained quadratic programming problem which can be efficiently solved on-line using, for instance, the Activeset Algorithm [20] and the Interior Point Method [22].

After obtaining the reference thrust of each rotor by solving the quadratic programming problem P2, the RPM command or PWM command can be subsequently calculated using a model obtained by propeller static thrust tests, which is omitted in this paper for readability.

\section{Simulation Validation}

\section{A. Case Study: Comparison Between P1 and P2 Allocation}

The proposed controller is first of all validated in a 6DoF simulation. The simulation platform uses the quadrotor model developed in [23], which takes complex aerodynamic effects into account. The quadrotor inertial and geometric parameters are given in Table. I. One of the innovations proposed in this article is utilizing P2 from 29) to replace P1 in (22), such that the undesirable angular rate $\tilde{\omega}$ can be suppressed. Fig. 5 shows $\tilde{\omega}$ and $n_{z}$ of two recovery maneuvers using P1 and P2 as allocation methods respectively. In both simulations, the failure of rotor 4 occurs when $\boldsymbol{n}=\left[\begin{array}{lll}-0.2, & 0.2, & 0.98\end{array}\right]^{T}$ and $\omega^{B}=\left[\begin{array}{ll}-15, & 15,0\end{array}\right]$. At this moment, the drone is almost upside down with a large $\tilde{\omega}$ at $17.3 \mathrm{rad} / \mathrm{s}$. The target thrust orientation of both are set as $\boldsymbol{n}_{\text {des }}=\left[\begin{array}{l}0,0,-1\end{array}\right]^{T}$, namely vertically upwards. It is clear that the trajectory with P2 allocation can effectively suppress $\tilde{\omega}$. Thereby the drone could recover its attitude within around $0.7 \mathrm{~s}$, whereas the same problem without restraining $\tilde{\omega}$ recovers at around $2 \mathrm{~s}$. 

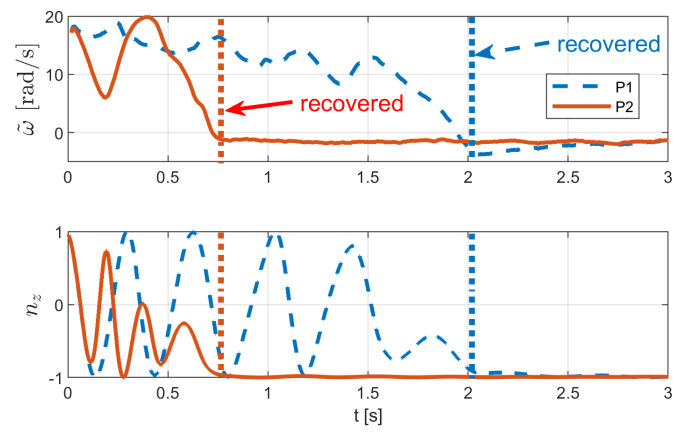

Fig. 5: Two trajectories initialized from the same condition while using different allocation methods. The upper plot shows the angular rates about the unrecoverable axis $\tilde{\omega}$. The method P2 can more effectively suppress $\tilde{\omega}$ than P1. The lower plot shows the vertical component of the thrust direction $\boldsymbol{n}$, namely $n_{z}$, which should converge to -1 when the vehicle thrust vector points upward. It is clear the method $\mathrm{P} 2$ results in much faster recovery speed than $\mathrm{P} 1$.

\section{B. Monte-Carlo Simulation}

A set of Monte-Carlo simulations are conducted to validate the proposed method. Another two methods are compared in these simulations: the method with P1 allocation, and the benchmark control method proposed by [12]. For each method, 200 trajectories are simulated from different initial conditions.

We simulate the scenario where the failure of rotor 4 happens during the forward flight at speed. The initial position and velocity of these flights are set as $\xi_{0}=[0,0,-50]^{T} \mathrm{~m}$, $\boldsymbol{v}_{0}=[10,0,0]^{T} \mathrm{~m} / \mathrm{s}$. The initial attitude is randomly selected in the entire $\mathrm{SO}(3)$, and the initial angular velocity $\omega_{0}^{B} \sim$ $U\left(-\omega_{0, \max }, \omega_{0, \max }\right)$ where $\omega_{0, \max }=[10,10,5]^{T} \mathrm{rad} / \mathrm{s}$.

The altitude time series of different methods are plotted in Fig. 6 And Fig. 7 shows the scatter plot of the initial conditions of these three methods with color showing the maximum height drop. For the benchmark method, there are 67 out of 200 flights crashed. Most of these crashed flights marked in red crosses concentrate in the area with positive initial $n_{z}$ which indicate downward pointing initial thrust orientations (Fig. 7. $\mathrm{a} 2$ ). On the other hand, the initial angular rates seem no special effect on the recovery performance. For method using P1 allocation shown in Fig. 6 b b and Fig. 77 $\mathrm{b}$, there are 4 crashes but many of the rest recover after dropping for a large amount of altitude. There are two crashes concentrate on the top-left $\omega_{x}-\omega_{y}$ plane of Fig. 77b1 meaning that these flights are initialized with large $\tilde{\omega}$. In comparison, the proposed controller using $\mathrm{P} 2$ allocation method recovers the damaged drone in all of the 200 flights. $95 \%$ of these flights could recover with a height drop of less than $10 \mathrm{~m}$ while only 1 flight recovers after dropping over $30 \mathrm{~m}$, as is shown in Fig. 6rc and Fig. 가c.

\section{EXPERIMENTAL VALIDATION}

The proposed method is also validated in the real flight environment. The tested platform is a modified Parrot Be-

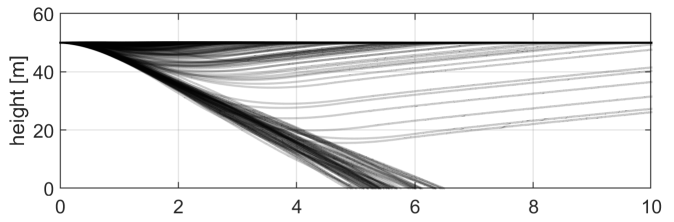

a.) $t[s]$

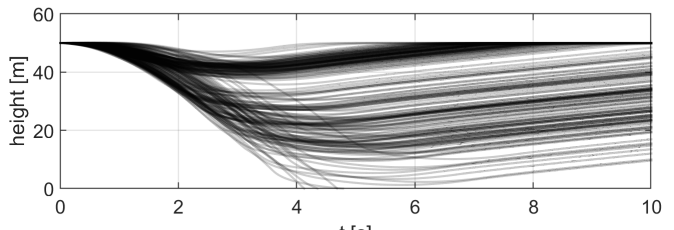

b.)

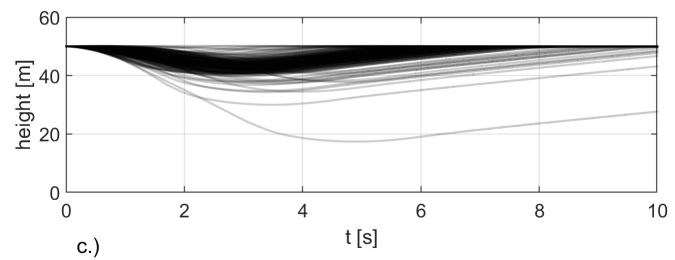

Fig. 6: Altitude time series of a set of Monte-Carlo simulation including 200 flights initialized from random attitude and angular velocities with different flight control methods. a.) The benchmark method. b.) The proposed method but using P1 allocation. c.) The proposed method.
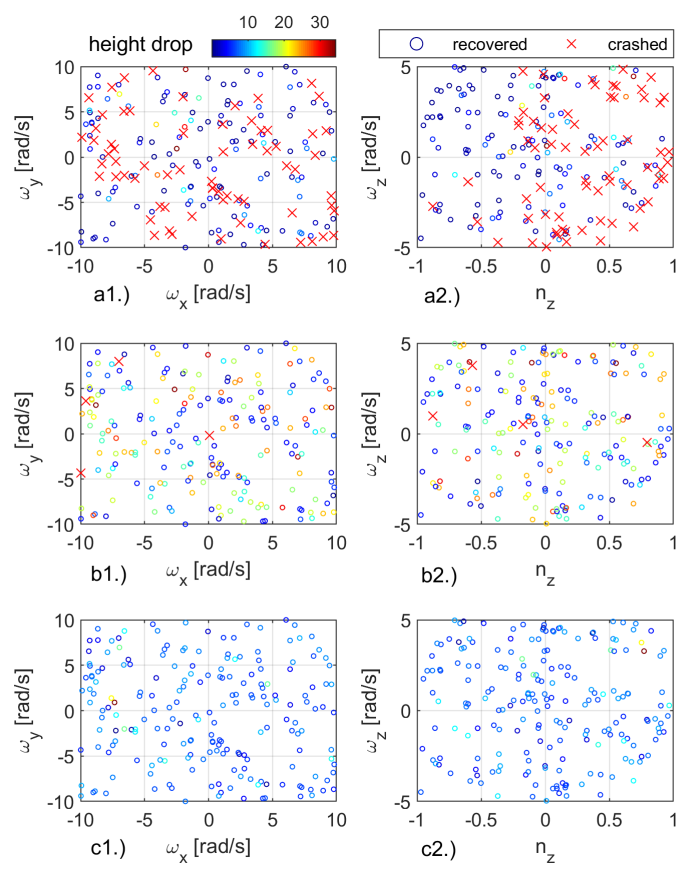

Fig. 7: Scatter plot of the initial conditions of the MonteCarlo simulation with colors showing the maximum height drop. The crashed flights are shown in red cross markers. a.) The benchmark method. b.) The proposed method but using P1 allocation. c.) The proposed method. 


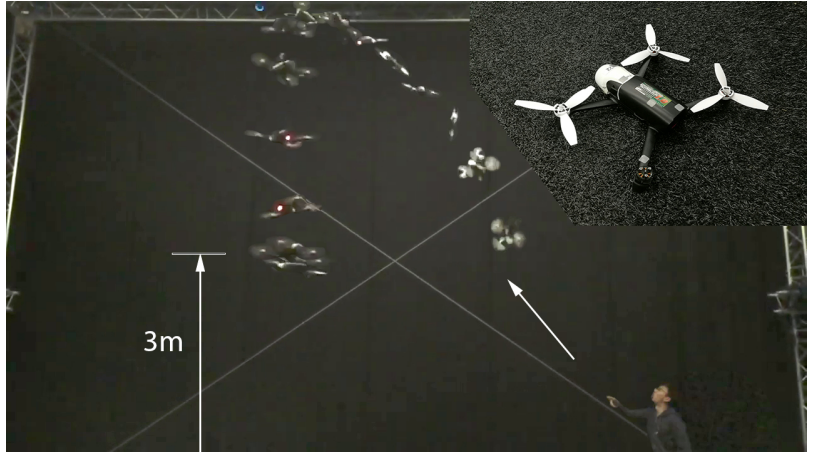

Fig. 8: Snapshots of the quadrotor recovery maneuver after being tossed into the air. The drone was finally stabilized at $3 \mathrm{~m}$ above the ground. Right top corner shows the photo of the tested quadrotor of which the left-back rotor was removed. (Video clip link: https://youtu.be/hrr2BzPLaMg)
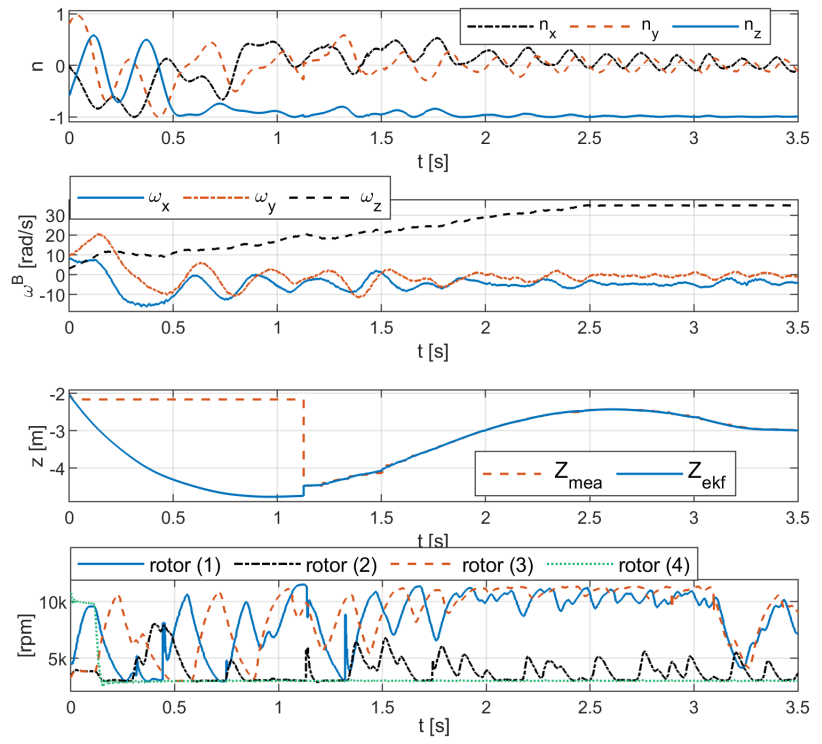

Fig. 9: Time history of the recovery maneuver. Subfigures from top to bottom present the thrust orientation $\boldsymbol{n}$, angular rates $\omega^{B}$, altitude $z$ and rotor speeds respectively.

bop 2 quadrotor, as Fig. 8 shows. The parameters of this quadrotor are given in Table. [1 The flight was conducted in the Cyberzoo, TU Delft where 12 cameras from the motion capturing system (Optitrack) measured the position of 6 reflective markers attached to the drone in $120 \mathrm{~Hz}$. The position information was then transmitted to the drone via $\mathrm{WiFi}$, and the controller was run on-board in $500 \mathrm{~Hz}$. The processor of the drone is a Parrot P7 dual-core CPU Cortex 9, and the IMU is MPU6050 for angular rate and specific force measurements.

To create the arbitrary initial condition, we threw the quadrotor with failure of rotor 4 into the air as Fig. 8 shows. After reaching an altitude of 2 meters, the drone started recovering. Fig. 9 shows the reduced attitude $\boldsymbol{n}$, the angular rates, height and the rotor RPM in the recovery process. The drone was finally recovered and stayed at $3 \mathrm{~m}$ over the
TABLE I: Inertial and geometric properties of the tested quadrotor.

\begin{tabular}{ccc}
\hline \hline parameter & value & unit \\
\hline $\boldsymbol{I}_{v}^{B}$ & $\operatorname{diag}(1.45,1.26,2.52) \times 10^{-3}$ & $\mathrm{kgm}^{2}$ \\
$m, l, \beta$ & $0.41,0.145,52.6$ & $\mathrm{~kg}, \mathrm{~m}, \mathrm{deg}$ \\
$s, \sigma$ & $1,0.01$ & - \\
\hline
\end{tabular}

TABLE II: Control parameters of the real-life flight test.

\begin{tabular}{cc|cc}
\hline \hline par. & value & par. & value \\
\hline $\boldsymbol{K}_{p, \text { pos }}$ & $\operatorname{diag}(1,1,15)$ & $\boldsymbol{K}_{p, \text { vel }}$ & $\operatorname{diag}(2,2,25)$ \\
$\boldsymbol{K}_{i \text {,vel }}$ & $\operatorname{diag}(1,1,5)$ & $\left(\theta_{1}, \theta_{2}\right)$ & $(30,70) \operatorname{deg}$ \\
$k_{p \text {, att }}$ & 8 & $\boldsymbol{K}_{p, \text { rate }}$ & $\operatorname{diag}(15,15,1)$ \\
$\boldsymbol{W}$ & $\operatorname{diag}\left(10^{4}, 10^{4}, 10^{2}, 4\right)$ & $\left(\lambda, \gamma, t_{h}, \tilde{\omega}_{\text {max }}\right)$ & $\left(0.1,10^{5}, 0.1,5\right)$ \\
\hline
\end{tabular}

ground with a fast yaw rate. The controller parameters of this set of the test are listed in Table. II

Since the motion capturing system is unable to measure the position of the drone with large attitude deviations from the hovering condition, an Extended Kalman Filter (EKF) is applied to fuse the camera measurements with the IMU measurements to obtain the position, velocity and attitude estimations. The 3rd subplot of Fig. 9 also shows EKF estimated altitude compared with the raw measurements and the latter keeps constant before $t=1.3 \mathrm{~s}$ due to loss of tracking of the reflective markers.

The in-door tests have a success rate of 71\% (46 out of 65 throws). However, those initialized from upside-down orientations and large $\tilde{\omega}$ is rather hard to recover before touching the ground. This is because of the height limitation of the laboratory (6 meters effective height) while it requires about 10 meters to completely recover from the upset condition. Therefore, out-door flight tests will be performed in future research, together with improved state estimation methods.

\section{CONCLUSIONS}

An upset recovery control method for a quadrotor with one rotor failure has been proposed and tested in this research. The controller can stabilize the quadrotor from arbitrary initial orientations and a wide range of angular velocities to the relaxed hovering condition. A novel control allocation approach is developed to suppress the undesirable angular velocities, which is important to the recovery performance. To demonstrate the reliability of the method, we have conducted Monte-Carlo simulations from random initial conditions. It has shown that the proposed method can timely recover the quadrotor with a height drop of less than $10 \mathrm{~m}$ in over $95 \%$ flights. In the real-flight test, the controller can recover the damaged quadrotor after being randomly tossed into the air. Further tests in outdoor environments, with onboard state estimation, are suggested for future research.

\section{ACKNOWLEDGEMENT}

The authors would like to thank Xuerui Wang and the MAVLab for their support during flight tests. 


\section{REFERENCES}

[1] J. K. Stolaroff, C. Samaras, E. R. O’Neill, A. Lubers, A. S. Mitchell, and D. Ceperley, "Energy use and life cycle greenhouse gas emissions of drones for commercial package delivery," Nature Communications, vol. 9, p. 409, dec 2018.

[2] T. Li, Y. Zhang, and B. W. Gordon, "Passive and active nonlinear fault-tolerant control of a quadrotor unmanned aerial vehicle based on the sliding mode control technique," Proceedings of the Institution of Mechanical Engineers, Part I: Journal of Systems and Control Engineering, vol. 227, pp. 12-23, jan 2013.

[3] X. Wang, S. Sun, E.-J. van Kampen, and Q. Chu, "Quadrotor Fault Tolerant Incremental Sliding Mode Control driven by Sliding Mode Disturbance Observers," Aerospace Science and Technology, vol. 87, pp. 417-430, 2019.

[4] A. Lanzon, A. Freddi, and S. Longhi, "Flight Control of a Quadrotor Vehicle Subsequent to a Rotor Failure," Journal of Guidance, Control, and Dynamics, vol. 37, pp. 580-591, mar 2014.

[5] M. W. Mueller and R. D'Andrea, "Stability and control of a quadrocopter despite the complete loss of one, two, or three propellers," in 2014 IEEE International Conference on Robotics and Automation (ICRA), pp. 45-52, IEEE, may 2014.

[6] M. W. Mueller and R. D'Andrea, "Relaxed hover solutions for multicopters: Application to algorithmic redundancy and novel vehicles," The International Journal of Robotics Research, vol. 35, pp. 873-889, jul 2016.

[7] V. Lippiello, F. Ruggiero, and D. Serra, "Emergency landing for a quadrotor in case of a propeller failure: A backstepping approach," in IEEE International Conference on Intelligent Robots and Systems, no. Iros, pp. 4782-4788, 2014.

[8] V. Lippiello, F. Ruggiero, and D. Serra, "Emergency landing for a quadrotor in case of a propeller failure: A PID Based Approach," in IEEE International Conference on Intelligent Robots and Systems, pp. 4782-4788, 2014.

[9] P. Lu and E.-J. van Kampen, "Active fault-tolerant control for quadrotors subjected to a complete rotor failure," in 2015 IEEE/RSJ International Conference on Intelligent Robots and Systems (IROS), pp. 4698-4703, IEEE, sep 2015.

[10] Z. Hou, P. Lu, and Z. Tu, "Nonsingular terminal sliding mode control for a quadrotor UAV with a total rotor failure," Aerospace Science and Technology, vol. 98, p. 105716, 2020.

[11] J. Stephan, L. Schmitt, and W. Fichter, "Linear Parameter-Varying Control for Quadrotors in Case of Complete Actuator Loss," Journal of Guidance, Control, and Dynamics, vol. 41, no. 10, pp. 2232-2246, 2018.

[12] S. Sun, L. Sijbers, X. Wang, and C. de Visser, "High-Speed Flight of Quadrotor Despite Loss of Single Rotor," IEEE Robotics and Automation Letters, vol. 3, pp. 3201-3207, oct 2018.

[13] L. Crespo, S. Kenny, D. Cox, and D. Murri, "Analysis of Control Strategies for Aircraft Flight Upset Recovery," in AIAA Guidance, Navigation, and Control Conference, (Reston, Virigina), American Institute of Aeronautics and Astronautics, aug 2012.

[14] J. A. Engelbrecht, S. J. Pauck, and I. K. Peddle, "A Multi-Mode Upset Recovery Flight Control System for Large Transport Aircraft," in AIAA Guidance, Navigation, and Control (GNC) Conference, (Reston, Virginia), American Institute of Aeronautics and Astronautics, aug 2013.

[15] C. M. Belcastro, "Aircraft loss of control: Analysis and requirements for future safety-critical systems and their validation," in ASCC 2011 - 8th Asian Control Conference - Final Program and Proceedings, pp. 399-406, 2011.

[16] A. A. Lambregts, G. Nesemeier, J. E. Wilborn, and R. L. Newman, "Airplane upsets: Old problem, new issues," AIAA Modeling and Simulation Technologies Conference and Exhibit, no. August, pp. 110, 2008.

[17] M. Faessler, F. Fontana, C. Forster, and D. Scaramuzza, "Automatic re-initialization and failure recovery for aggressive flight with a monocular vision-based quadrotor,' Proceedings - IEEE International Conference on Robotics and Automation, vol. 2015-June, no. June, pp. 1722-1729, 2015.

[18] R. Mahony, V. Kumar, and P. Corke, "Multirotor Aerial Vehicles: Modeling, Estimation, and Control of Quadrotor," IEEE Robotics \& Automation Magazine, vol. 19, pp. 20-32, sep 2012.

[19] P. W. Fortescue and G. G. Swinerd, "Rigid-Body Attitude Control," IEEE Control Systems, vol. 31, pp. 30-51, jun 2011.

[20] J. Petersen and M. Bodson, "Constrained quadratic programming techniques for control allocation," IEEE Transactions on Control Systems Technology, vol. 14, pp. 91-98, jan 2006.

[21] A. . Smeur, E. . Höppener, and D. Wagter, "Prioritized Control Allocation for Quadrotors Subject to Saturation," in International Micro Air Vehicle Conference and Flight Competition, no. September, (Moschetta, G. Hattenberger), pp. 37-43, 2017.

[22] R. J. Vanderbei, "LOQO: An interior point code for quadratic programming," Optimization Methods and Software, vol. 11, no. 1, pp. 451484, 1999.

[23] S. Sun and C. de Visser, "Aerodynamic Model Identification of a Quadrotor Subjected to Rotor Failures in the High-Speed Flight Regime," IEEE Robotics and Automation Letters, vol. 4, pp. 38683875 , oct 2019. 